\title{
Size-dependent Photovoltaic Properties of Solar Cells Containing Si Quantum Dots/SiC Multilayers
}

\author{
Yunqing $\mathrm{Cao}^{1,2}$, , Dong $\mathrm{Wu}^{2}$, Ping $\mathrm{Zhu}^{2}$, Zhaoyun $\mathrm{Ge}^{1,3}$, Wei $\mathrm{Li}^{1}$, Jun $\mathrm{Xu}^{1}$, Kunji Chen ${ }^{1}$ \\ ${ }^{1}$ National Laboratory of Solid State Microstructures and School of Electronic Science and Engineering and \\ Collaborative Innovation Center of Advanced Microstructures, Nanjing University, Nanjing 210093, China \\ ${ }^{2}$ College of Physical Science and Technology, Yangzhou University, Yangzhou 225009, China \\ ${ }^{3}$ School of Science, Jiangsu University of Science and Technology, Zhenjiang 212003, China \\ *Corresponding author. E-mail: yqcao@yzu.edu.cn
}

\begin{abstract}
Recently, many kinds of Si nanostructures have been extensively investigated, in which, Si quantum dot (Si QD) is one of the potential candidates for all-Si tandem solar cells. Because the optical bandgap of Si QDs can be tunable via size controlling, it can match the solar spectrum in a wide range and consequently improve the spectral response. In this work, $\mathrm{Si} \mathrm{QDs} / \mathrm{SiC}$ multilayers with controllable dot sizes were fabricated and characterized. The Raman spectra and transmission electron microscopy (TEM) observation revealed the formation of size-controllable Si QDs. The absorption measurement showed that the bandgap of Si QDs was red shifted to the long wavelength range with the dot size increasing, which agrees well with the quantum confinement effect. Moreover, heterojunction solar cells containing different sized-Si QDs/SiC multilayers were proposed and investigated. The solar cells exhibited strong size-dependent photovoltaic properties and the best cell had the power conversion efficiency (PCE) of 7.27\%. Furthermore, the external quantum efficiency (EQE) measurement demonstrated the Si QDs contribution of light absorption and response in ultraviolet-visible range, which provides a promising way to realize better spectral match by applying different sized-Si QDs in the future photovoltaic devices.
\end{abstract}

Key words: Si quantum dots (Si QDs); Silicon carbide (SiC); Multilayers (MLs); Solar cell; Sizedependent photovoltaic properties 


\section{Introduction}

\section{Methods}

The a-Si:H/SiC MLs were fabricated on quartz and p-Si substrates in PECVD system by alternatively repeating the a-Si deposition and the a-SiC deposition processes. The radio frequency power was $30 \mathrm{~W}$ and the substrate temperature was kept at $250^{\circ} \mathrm{C}$ during the deposition process. The a-Si:H sublayer was deposited by using pure silane $\left(\mathrm{SiH}_{4}\right)$, while the a-SiC sublayer was deposited by using a gas mixture of $\mathrm{SiH}_{4}$ and methane $\left(\mathrm{CH}_{4}\right)$ with the gas ratio $\mathrm{R}\left(\mathrm{R}=\left[\mathrm{CH}_{4}\right] /\left[\mathrm{SiH}_{4}\right]\right)$ of 10. The thickness of a-Si:H sublayer varied from $2 \mathrm{~nm}$ to $8 \mathrm{~nm}$ by changing the deposition time, and the thickness of a-SiC sublayer was kept at $2 \mathrm{~nm}$. For better comparison, the total thickness of a-Si:H layers was kept the same, so the periodicity was 12, 6 and 3 for the samples with a-Si:H sublayer thickness of $2 \mathrm{~nm}, 4 \mathrm{~nm}$ and $8 \mathrm{~nm}$, respectively. The post-treatment performed in $\mathrm{N}_{2}$ atmosphere includes two steps, which is dehydrogenation at $450^{\circ} \mathrm{C}$ for $1 \mathrm{~h}$ and subsequently annealing at $900^{\circ} \mathrm{C}$ for $1 \mathrm{~h}$. The structural change of the Si/SiC MLs before and after annealing was evaluated by Raman spectroscopy (Jobin Yvon Horiba HR800 spectrometer). The formation of Si QDs was determined by TEM observation using Technai G2 operated at $200 \mathrm{KV}$. The optical absorption of the $\mathrm{Si} / \mathrm{SiC}$ MLs was measured at room temperature by Shimadzu UV-3600 spectrophotometer.

In order to investigate the size-dependent photovoltaic properties of present samples, we fabricated Si QDs/SiC MLs including different sized-Si QDs on p-type and heavily doped p-type ( $\mathrm{p}^{+}$-type) $\mathrm{Si}$ wafers, followed by phosphorus-doped amorphous $\mathrm{Si}$ layer deposition on the multilayers to get p-i-n (or $\left.\mathrm{p}^{+}-\mathrm{i}-\mathrm{n}\right)$ heterojunction structures. The Si wafers were additionally dipped in $2 \% \mathrm{HF}$ solution for $10 \mathrm{~s}$ to remove the native surface oxide before the fabrication process. Finally, Al electrode was evaporated on the surface and rear side of Si wafers and solar cell devices were achieved. The illuminated current density-voltage $(J-V)$ characteristics of the solar cells containing different sized-Si QDs/SiC MLs were measured under an AM1.5 (100 mW/ $\left.\mathrm{cm}^{2}\right)$ illumination by using a Keithley 610C electrometer. The external quantum efficiency (EQE) spectra were collected by the QEX-10 spectral response measurement system in the wavelength range of $300 \mathrm{~nm}-1200$ nm.

\section{Results and Discussion}




\subsection{Structural Characterizations of $\mathrm{Si} \mathrm{QDs} / \mathrm{SiC}$ MLs}

Figs. 1a-c are the cross-sectional TEM image of as-deposited a-Si (2nm)/a-SiC (2nm) MLs, a-Si $(4 \mathrm{~nm}) / \mathrm{a}-\mathrm{SiC}(2 \mathrm{~nm}) \mathrm{MLs}$ and a-Si $(8 \mathrm{~nm}) / \mathrm{a}-\mathrm{SiC}(2 \mathrm{~nm})$ MLs. The layered structures and smooth interfaces of $\mathrm{Si} / \mathrm{SiC}$ MLs can be clearly identified. The thickness of a-SiC sublayers is $1.9 \mathrm{~nm}$ and the thickness of a-Si sublayers is $2.1 \mathrm{~nm}, 4.2 \mathrm{~nm}$ and $8.2 \mathrm{~nm}$, respectively, which is very close to the pre-designed value estimated from the deposition rule. Fig. 2 shows the Raman spectra of $900^{\circ} \mathrm{C}$ annealed $\mathrm{Si} / \mathrm{SiC}$ MLs samples. An intense and sharp peak appears in the Raman spectrum around $520 \mathrm{~cm}^{-1}$ for all the samples, which indicates that the nano-crystallized Si has been formed in the aSi sublayer. With the thickness of the a-Si sublayer increasing, the Raman peak shifted gradually towards $520 \mathrm{~cm}^{-1}$, which demonstrates that the size of Si QDs is enlarged with increasing the a-Si sublayer thickness. We fitted the Raman spectrum via the Gaussian deconvolution by two components, which is located at $480 \mathrm{~cm}^{-1}$ (corresponding to the TO mode of amorphous $\mathrm{Si}$ ) and 520 $\mathrm{cm}^{-1}$ (corresponding to the TO mode of nano-crystallized $\mathrm{Si}$ ). The crystallinity ratio $\left(\mathrm{X}_{\mathrm{c}}\right)$ is figured out as $36.8 \%, 49.5 \%$ and $52.8 \%$ by integrated Gaussian peaks of $520 \mathrm{~cm}^{-1}$ and $480 \mathrm{~cm}^{-1}$ [24]. According to the phonon confinement model [25], the average size of Si QDs is estimated to be 3.0 $\mathrm{nm}, 4.8 \mathrm{~nm}$ and $10.5 \mathrm{~nm}$, respectively. Figs. $1 \mathrm{~d}-\mathrm{f}$ are the cross-sectional TEM images of the $900^{\circ} \mathrm{C}$ annealed multilayered samples, which exhibits the formed Si QDs after annealing. The size of Si QDs is observed to be $2.5 \mathrm{~nm}, 5.0 \mathrm{~nm}$ and $8.9 \mathrm{~nm}$, which is in good agreement with the Raman evaluation. By the Raman and TEM characterization, it is found that the Si QDs size can be well constrained by the $\mathrm{Si} \mathrm{QDs} / \mathrm{SiC}$ MLs during the deposition and high temperature annealing process, which suggests that the Si QDs size can be precisely controlled by varying the thickness of a-Si sublayers in our fabrication method. 


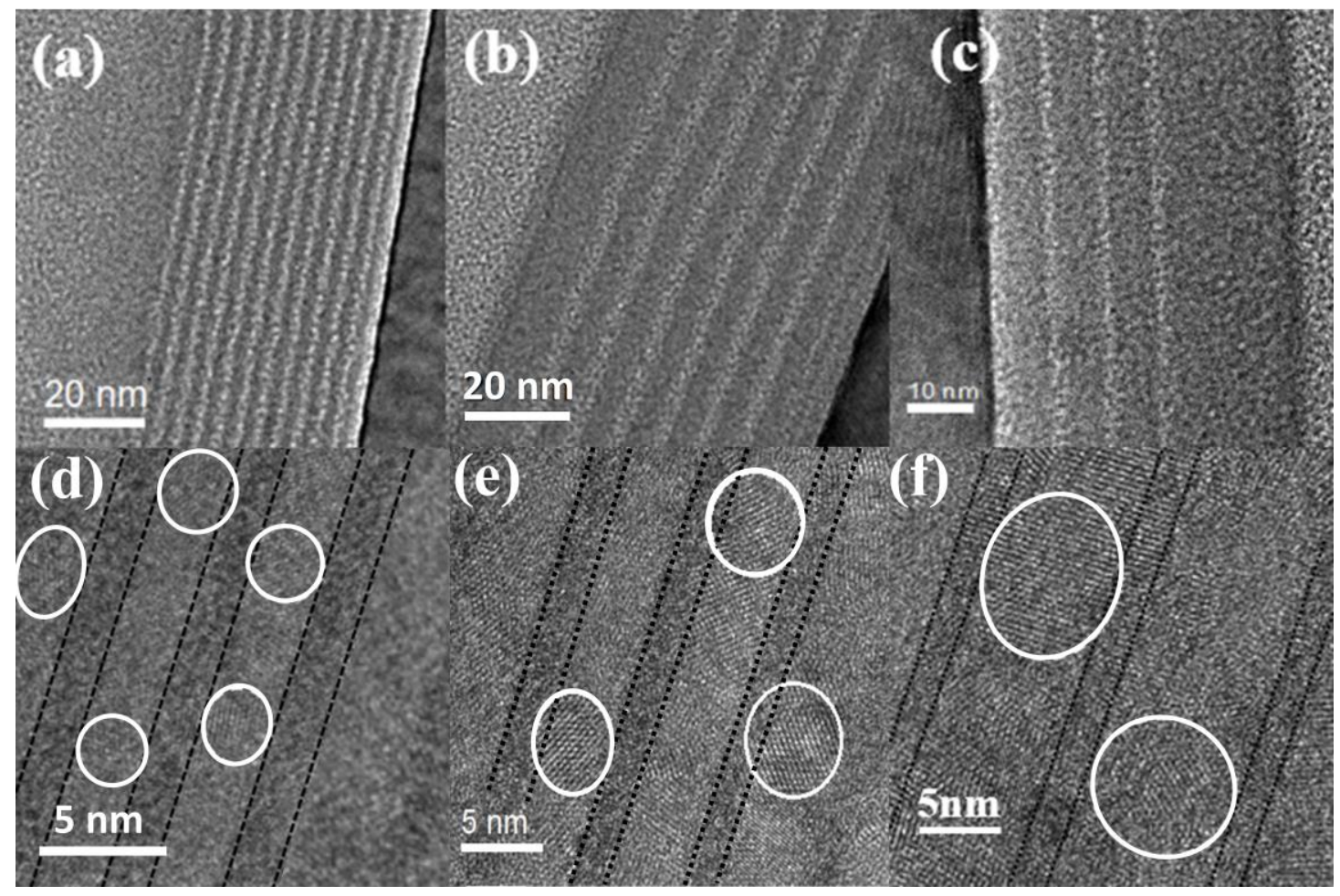

Figure 1 Cross-sectional TEM images of as-deposited (a) a-Si (2nm)/a-SiC(2nm) MLs; (b) a-Si (4nm)/a-SiC(2nm) MLs; (c) a-Si (8nm)/a-SiC(2nm) MLs. The TEM images of annealed (d) Si QDs (2nm)/ SiC(2nm) MLs; (e) Si QDs (4nm)/ SiC(2nm) MLs; (f) Si QDs (8nm)/ SiC(2nm) MLs.

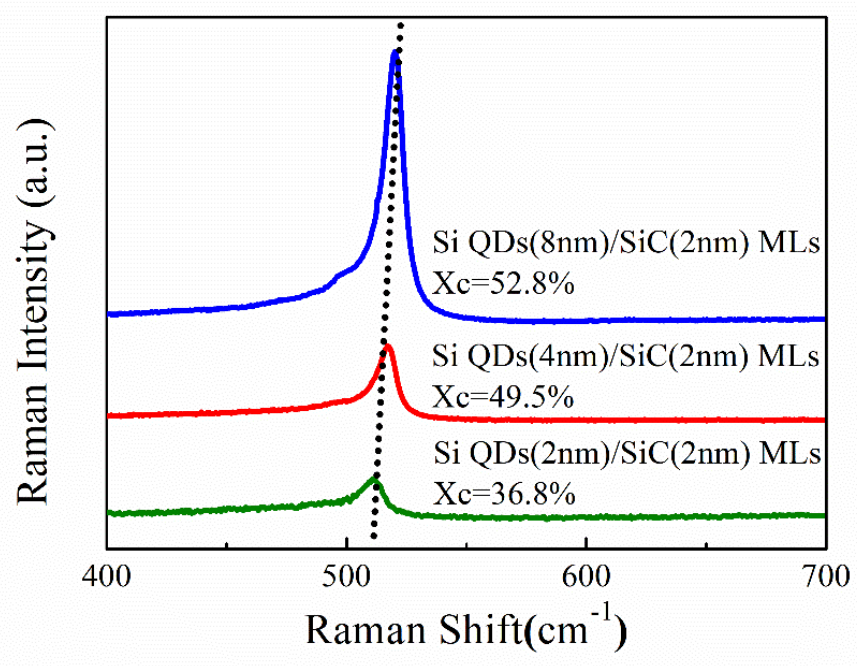

Figure 2 Raman spectra of $900^{\circ} \mathrm{C}$ annealed Si QDs/SiC MLs.

\subsection{Optical properties of $\mathrm{Si}$ QDs/SiC MLs}

In order to study the optical properties of the different sized-Si QDs/SiC MLs, we measured the transmission and reflection of those samples. The optical absorption coefficient $\alpha$ in the spectral 
range of $300 \mathrm{~nm}-800 \mathrm{~nm}$ is deduced and shown in Fig. 3. In our previous work, the absorption of Si QDs/SiC MLs was almost unchanged by changing the thickness of SiC layers, which indicates that the Si QDs play the dominant role in optical absorption [26]. As shown in Fig. 3, the optical absorption edge is shifted to the long wavelength region by increasing the Si QDs size. The red shifting behavior of the Si QDs optical absorption was already observed and discussed in $\mathrm{Si}$ QDs/SiO 2 MLs, Si QDs/SiN $\mathrm{SL}_{\mathrm{x}}$ MLs and $\mathrm{Si}$ QDs/SiC MLs in our previous work [27-29], and it was demonstrated to result from the quantum confinement effect [30]. The optical bandgap $\left(\mathrm{Eg}_{\mathrm{opt}}\right)$ can be extracted from Tauc's function. The deduced $\mathrm{Eg}_{\mathrm{opt}}$ of $\mathrm{Si} \mathrm{QDs} / \mathrm{SiC} \mathrm{MLs}$ is $2.0 \mathrm{eV}, 1.5 \mathrm{eV}$ and 1.2 $\mathrm{eV}$, respectively, as the dot size is $2.5 \mathrm{~nm}, 5.0 \mathrm{~nm}$ and $8.9 \mathrm{~nm}$. As we discussed before, we developed a modified effective mass approximation (EMA) model to estimate the optical band gap of $\mathrm{Si}$ QDs/SiC MLs instead of an infinite barrier model by considering the Coulomb effect and the correlation energy terms [31]. Based on this model, the calculated $\mathrm{Eg}_{\mathrm{opt}}$ results are consistent with the experimental results. It is worth noting that for Si QDs with $2.5 \mathrm{~nm}$ and $5.0 \mathrm{~nm}$, the $\mathrm{Eg}_{\mathrm{opt}}$ is about $2.0 \mathrm{eV}$ and $1.5 \mathrm{eV}$, which is the theoretically expected bandgap of the top-cell and middle-cell materials in a 3-juction tandem cell [6]. Moreover, since it is demonstrated that the optical bandgap can be tunable by changing the size of Si QDs due to the quantum confinement effect, the $\mathrm{Si}$ QDs/SiC multilayered structure is a good choice for future multi-junction tandem solar cell devices requiring bandgap controllable materials.

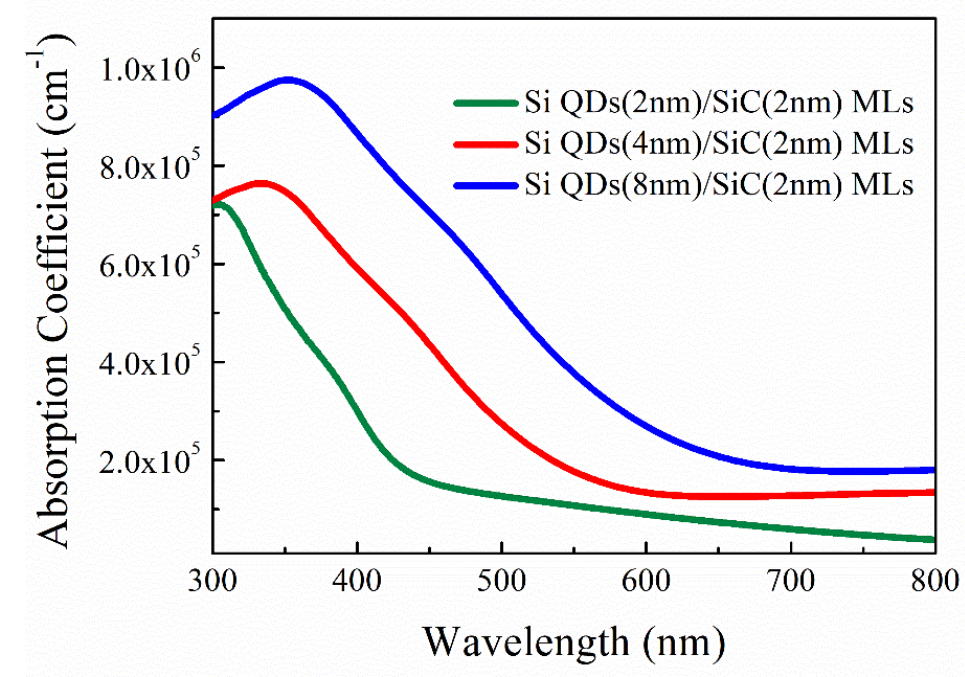

Figure 3 Optical absorption coefficient spectra of Si QDs/SiC MLs. 


\subsection{Size-dependent photovoltaic properties of solar cells containing Si QDs/SiC MLs}

To study the electronic properties of the solar cell samples, we measured the dark current-voltage $(I-V)$ relationship and illuminated $J-V$ characteristics. The dark $I-V$ characteristics of different sized$\mathrm{Si}$ QDs/SiC MLs based devices are shown in Fig. 4. The rectification ratio at $\pm 1 \mathrm{~V}$ is above $10^{2}$ for all the samples, which indicates the well-formed p-i-n structures in our case. The $I-V$ characteristics can be divided by 3 parts. When $\mathrm{V}<0.4 \mathrm{~V}$ (region 1 ), the $I-V$ relationship is linearly dependent on the shunt resistor $\left(\mathrm{R}_{\text {sh }}\right)$. When $0.4 \mathrm{~V}<\mathrm{V}<0.6 \mathrm{~V}$ (region 2), the current increases exponentially with the forward voltage, which presents the diode behavior. When $\mathrm{V}>0.6 \mathrm{~V}$ (region 3), the $I-V$ characteristics deviated from the ideal diode behavior, which should be attributed to the spacecharge-limited current [32]. In region 2, the $I-V$ curves can be fitted by using a standard diode relationship I $\propto \exp (\mathrm{qV} / \mathrm{nkT})$ The fitted ideality factor $\mathrm{n}$ is $2 \sim 3$ for all the Si QDs/SiC MLs based devices, which indicates that the carrier tunneling is the dominant current transport mechanism. For the $\mathrm{Si} \mathrm{QDs} / \mathrm{SiC}$ stack model, the carrier tunneling probability $(\mathrm{Te})$ is given as Equation $1[5,33]$

$$
T_{e}=16 \exp \left(-d \sqrt{\frac{8 m^{*}}{\hbar^{2}} \Delta E}\right)
$$

where $d$ is the barrier width, $m *$ is the effective mass of electrons or holes, $\Delta E$ is the barrier height between Si QDs and SiC. As discussed above, with the dot size decreasing, the bandgap of Si QDs is broadened correspondingly, leading to a lower tunneling barrier height $\Delta E$ in Equation 1. Since the barrier width $d$ keeps the same for all the $\mathrm{Si} \mathrm{QDs} / \mathrm{SiC}$ multilayered structures, $T_{e}$ will increase monotonically when the Si QDs size decreases, which means more electrons and holes can tunnel through the $\mathrm{SiC}$ barrier and then be collected by the electrodes. As a result, the dark current increases when Si QDs size decreases, which is shown in Fig. 4. 


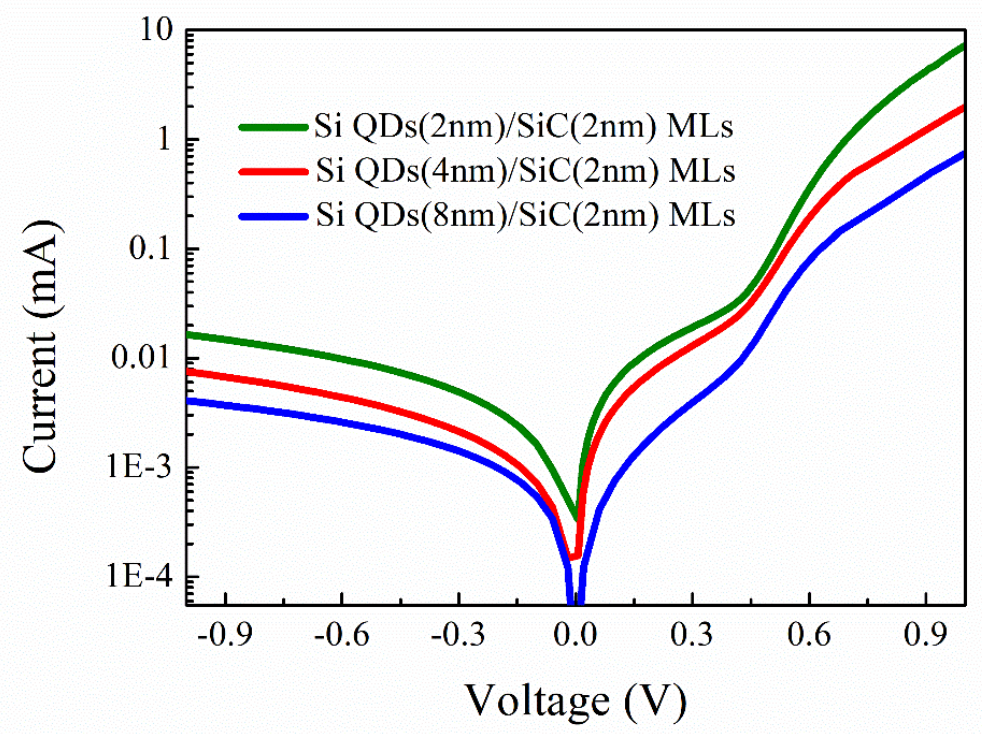

Figure 4 Dark current-voltage curves of solar cells containing different sized-Si QDs/SiC MLs.

Fig. 5 shows the AM1.5 illuminated $J-V$ curves of solar cells containing different sized-Si QDs/SiC MLs. The cell area is about $0.8 \mathrm{~cm}^{2}$. The device parameters including $V_{o c}, J_{s c}$, fill factor (FF) and PCE are summarized in Table. 1. Since the device performance of solar cells strongly depends on the photo-generated carrier tunneling and collection efficiency, the solar cell containing $2 \mathrm{~nm}$ sized-Si QDs shows the highest $J_{s c}$. However, in our experimental design, to keep the active layer thickness of the cells with smaller sizes of QDs means to increase the periodicity of MLs. The Si QDs (2 nm)/SiC MLs has the largest periodicity of 12 . The corresponding cell exhibits the lowest $V_{o c}$ due to the strong recombination in the interface states introduced by increasing the layer number. In addition, the contact resistance of the solar cell is increased with the number of $\mathrm{SiC}$ sublayers increasing, which will lead to the reduction of FF. Consequently, the PCE of solar cell containing 2 $\mathrm{nm}$ sized-Si QDs is only 4.59\%. In our case, all the impacts mentioned above are taken into consideration and optimized. When the thickness of Si QDs sublayer is $4 \mathrm{~nm}$, the solar cell shows the best performance with PCE of $7.27 \%$. 


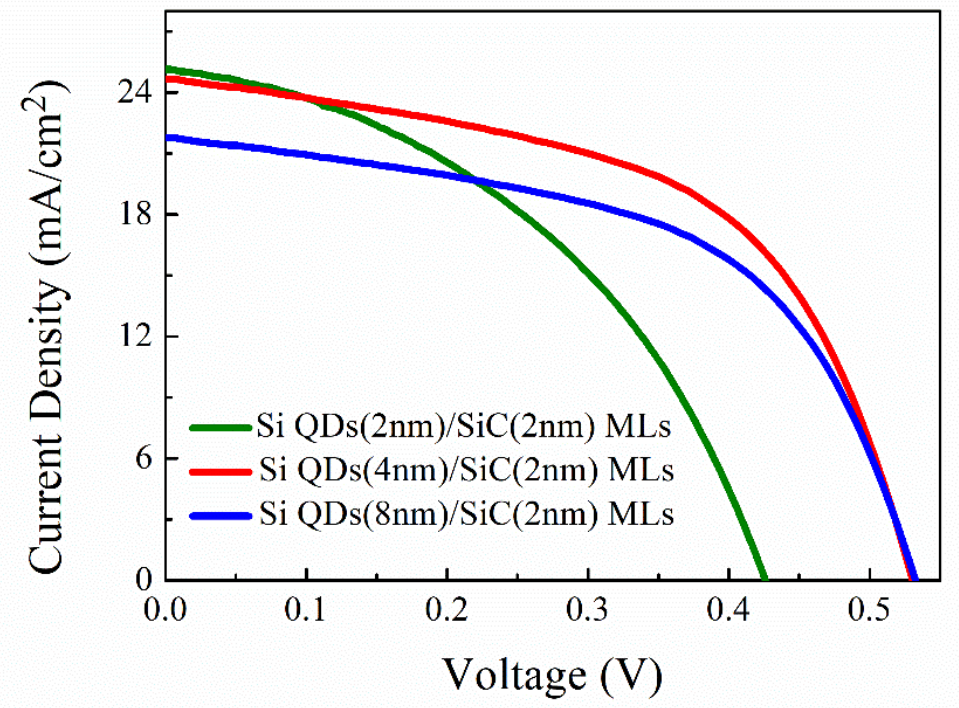

Figure 5 AM1.5 illuminated current density-voltage curves of solar cells containing different sized-Si QDs/SiC

MLs.

Table 1 The device parameters of solar cells containing different sized-Si QDs/SiC MLs.

\begin{tabular}{lcccc}
\hline & $V_{\text {oc }}(\mathbf{m V})$ & $\mathbf{J}_{\text {sc }}\left(\mathbf{m A} / \mathbf{c m}^{2}\right)$ & FF (\%) & PCE (\%) \\
\hline Si QDs(2nm)/SiC(2nm) MLs & 425 & 25.02 & 43.2 & 4.59 \\
Si QDs(4nm)/SiC(2nm) MLs & 530 & 24.66 & 55.6 & 7.27 \\
Si QDs(8nm)/SiC(2nm) MLs & 532 & 21.75 & 55.8 & 6.45 \\
\hline
\end{tabular}

Fig. 6 is the EQE of solar cells containing different sized-Si QDs/SiC MLs. The spectral response wavelength is red shifted to the long wavelength region with enlarging the Si QDs size. For example, at the short wavelength around $500 \mathrm{~nm}$, the EQE decreases about $20 \%$ with enlarging the size of $\mathrm{Si}$ QDs from $2 \mathrm{~nm}$ to $8 \mathrm{~nm}$. The main reason is the spectral mismatch and poorer response in the short wavelength region for the larger Si QDs. 


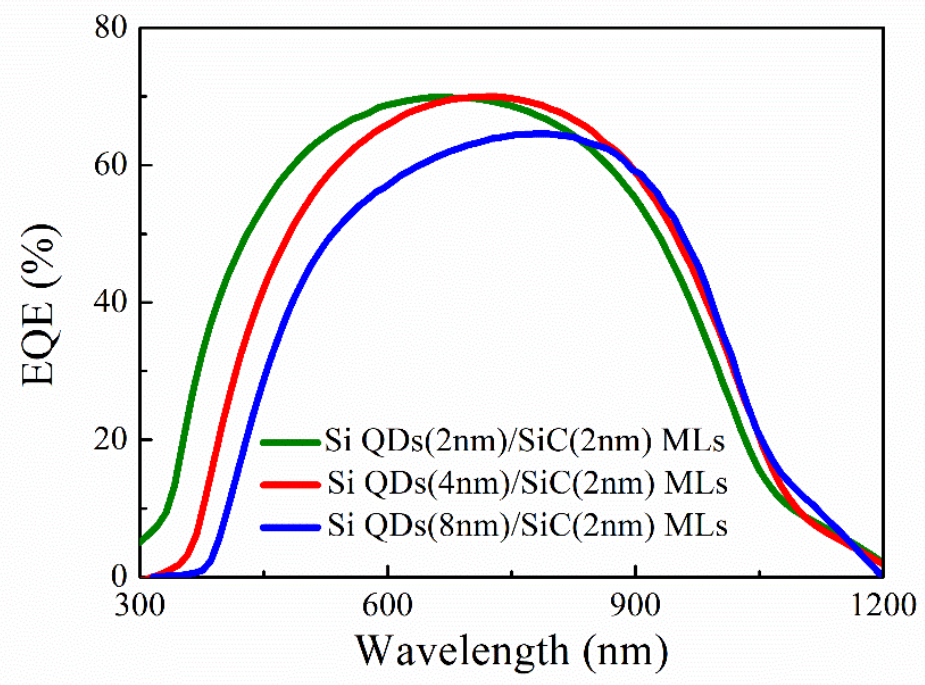

Figure 6 External quantum efficiency of solar cells containing different sized-Si QDs/SiC MLs.

However, as we discussed before, most of the incident light in this type of solar cell devices is absorbed in the c-Si substrates, which is revealed in the EQE results [34]. In order to exclude the absorption of c-Si substrates and further investigate the Si QDs contribution of spectral response, we used heavily doped $\mathrm{p}$ type $\left(\mathrm{p}^{+}\right) \mathrm{Si}$ substrates instead of general Si substrates to get solar cell structures. It is known that heavily doped semiconductors always act as "death layers" in solar cells, because they do not contribute to photo-generated carriers, due to the short minority-carrier lifetime and high recombination rate [35]. Fig. 7 shows the EQE of $\mathrm{p}^{+}-\mathrm{i}-\mathrm{n}$ device structures containing different sized-Si QDs/SiC MLs. The EQE is very low at the near infrared region (NIR) from 800 $\mathrm{nm}$ to $1200 \mathrm{~nm}$. This is because long wavelength light absorbed in the heavily doped substrates cannot generate photocurrent. Meanwhile, three peaks of EQE spectra located at $460 \mathrm{~nm}, 500 \mathrm{~nm}$ and $530 \mathrm{~nm}$, were observed for the devices with the Si QDs size of $2 \mathrm{~nm}, 4 \mathrm{~nm}$ and $8 \mathrm{~nm}$, respectively. It shows that the spectral response is red shifted to the long wavelength region by increasing the $\mathrm{Si}$ QDs size, which can be attributed to the reducing bandgap of Si QDs owing to the quantum confinement effect. Based on $\mathrm{EQE}$ results, the $J_{s c}$ contributed from cell spectral response in different wavelength range can be calculated according to Equation 2

$$
J_{s c}=\int_{\lambda_{1}}^{\lambda_{2}} \frac{F(\lambda) \cdot E Q E(\lambda)}{E(\lambda)} d \lambda
$$

where $F(\lambda)$ and $E(\lambda)$ is the flux of incident light and photon energy at the wavelength of $\lambda$. The 
integrated current density of the devices with the Si QDs size of $2 \mathrm{~nm}, 4 \mathrm{~nm}$ and $8 \mathrm{~nm}$ is 9.19 $\mathrm{mA} / \mathrm{cm}^{2}, 8.65 \mathrm{~mA} / \mathrm{cm}^{2}$ and $7.32 \mathrm{~mA} / \mathrm{cm}^{2}$, respectively, which can be attributed to the spectral response of Si QDs. Our present work systematically studies and demonstrates the size-dependent photovoltaic properties of solar cell devices containing different sized-Si QDs/SiC MLs, which provides a potential application of the Si QDs/SiC MLs in the future all-Si tandem solar cells.

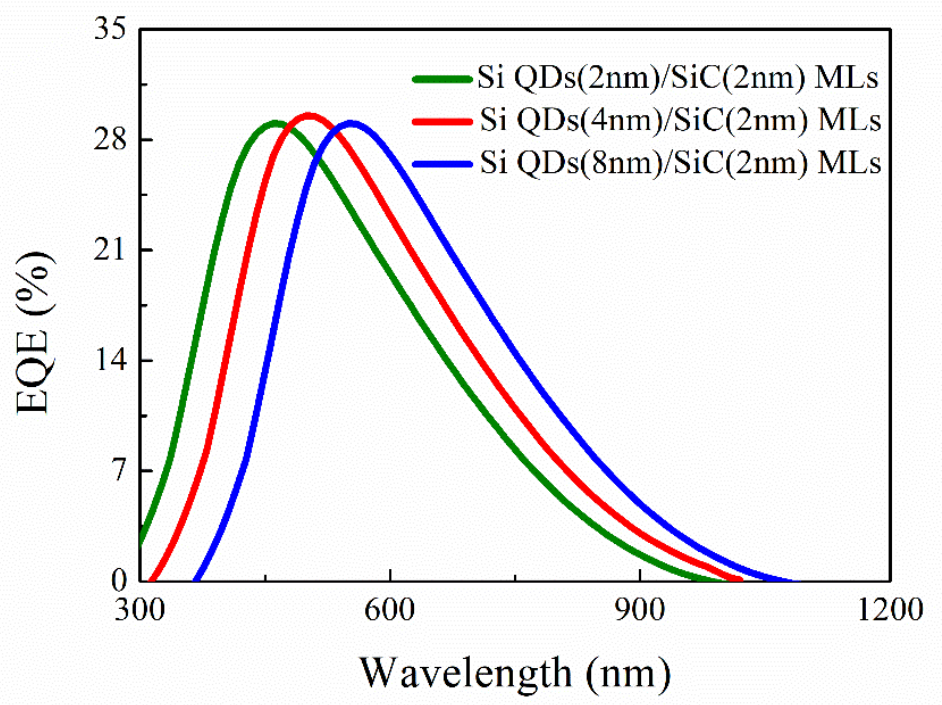

Figure 7 External quantum efficiency of $\mathrm{p}^{+}-\mathrm{i}-\mathrm{n}$ device structures containing different sized-Si QDs/SiC MLs.

\section{Conclusions}

In this work, we fabricated $\mathrm{Si}$ QDs/SiC stacked structures by annealing a-Si:H/SiC MLs at $900^{\circ} \mathrm{C}$. The thickness of a-Si:H was designed to be $2 \mathrm{~nm}, 4 \mathrm{~nm}$ and $8 \mathrm{~nm}$ and thickness of amorphous $\mathrm{SiC}$ layer was kept at 2nm. Raman spectra and TEM observation revealed that the Si QDs can be formed after annealing and the crystallinity was about $36.8 \%, 49.5 \%$ and $52.8 \%$, respectively. The Si QDs with diameters of $2.5 \mathrm{~nm}, 5.0 \mathrm{~nm}$ and $8.9 \mathrm{~nm}$ were successfully obtained, which means the size of Si QDs can be well controlled. We studied the optical properties of the Si QDs/SiC MLs and found that the optical absorption edge was red shifted to the long wavelength region by increasing the $\mathrm{Si}$ QDs size, which could be attributed to the quantum confinement effect. Moreover, we fabricated the different sized-Si QDs/SiC MLs together with phosphorus-doped a-Si films on p-Si substrates to get heterojunction solar cell devices. It was found that the photovoltaic performances showed strong dependence on Si QD sizes, and the best photovoltaic parameters were $\mathrm{V}_{\mathrm{oc}}$ of $530 \mathrm{mV}, \mathrm{J}_{\mathrm{sc}}$ of $24.66 \mathrm{~mA} / \mathrm{cm}^{2}$, and PCE of 7.27\% from solar cell containing Si QDs (4 nm)/SiC (2 nm) MLs. 
Furthermore, the EQE measurement of $\mathrm{p}^{+}-\mathrm{i}-\mathrm{n}$ structures, which excluded the absorption of $\mathrm{c}-\mathrm{Si}$ substrates, clearly demonstrated the contribution of Si QDs in the spectral response in ultravioletvisible light region. The spectral response in short wavelength range was enhanced with decreasing the Si QDs size from $8 \mathrm{~nm}$ to $2 \mathrm{~nm}$, which reveals the size-dependent photovoltaic properties of solar cells containing Si QDs/SiC MLs. Our experiment results infer that the different sized-Si QDs/SiC MLs can be used as a potential candidate to get better spectral match for advanced optoelectronic devices.

\section{Abbreviations}

Si QDs/SiC MLs: Si quantum dots/SiC multilayers; $\mathrm{V}_{\mathrm{oc}}$ : open circuit voltage; $\mathrm{J}_{\mathrm{sc}}$ : short circuit current density; PCE: power conversion efficiency; FF: fill factor; EQE: external quantum efficiency.

\section{Availability of data and materials}

The datasets used during the current study are available from the corresponding author of this article.

\section{Competing interests}

The authors declare that they have no competing interests.

\section{Funding}

This research was funded by NSFC (No. 61704148), NSF of Jiangsu Province (No. BK20170514), NSF of Jiangsu Higher Education Institutions (No. 17KJB140030), NSF of Yangzhou City (No. YZ2017102) and Open project of state key laboratory of solid state microstructure physics (No. M30039).

\section{Authors' contributions}

YQC and JX conceived the idea and carried out the experiments. YQC, DW and WL participated in the preparation of the samples. YQC, DW, PZ and ZYG took part in the experiments and the discussion of the results. YQC drafted the manuscript with the instruction of JX and KJC. All authors read and approved the final manuscript.

\section{Acknowledgments}

Not applicable 


\section{References}

[1] Shockley W, Queisser HJ. Detailed balance limit of efficiency of p-n junction solar cells. J Appl Phys 1961, 32:510-519.

[2] Green MA. Third Generation Photovoltaics: Advanced Solar Energy Conversion, Springer, Berlin, 2003, 35-66.

[3] Yamamoto A, Tsujino M, Ohkubo M, Hashimoto A. Metalorganic chemical vapor deposition growth of InN for InN/Si tandem solar cell. Sol. Energy Mater. Sol. Cells 1994, 35:53-60.

[4] Lunardi MM, Moore S, Alvarez-Gaitan JP, Yan C, Hao X J, Corkish R. A comparative life cycle assessment of chalcogenide/Si tandem solar modules. Energy 2018, 145: 700-709.

[5] Conibeer G, Green MA, Cho EC, Konig D, Cho YH, Fangsuwannarak T, Scardera G, Pink E, Huang YD, Puzzer T, Huang SJ, Song DY, Flynn C, Park S, Hao XJ, Mansfield D. Silicon quantum dot nanostructures for tandem photovoltaic cells. Thin Solid Films 2008, 516: 6748-6756.

[6] Meillaud F, Shah A, Droz C, Vallat-Sauvain E, Miazza C. Efficiency limits for single-junction and tandem solar cells. Sol. Energy Mater. Sol. Cells 2006, 90: 2952-2959.

[7] Baba M, Makita K, Mizuno H, Takato H, Sugaya T, Yamada N. Effect of series resistances on conversion efficiency of GaAs/Si tandem solar cells with areal current-matching technique. IEEE J. Photovolt. 2018, 8: $654-660$.

[8] Hajijafarassar A, Martinho F, Stulen F, Grini S, López-Mariño S, Espíndola-Rodrí guez M, Döbeli M, Canulescu S, Stamate E, Gansukh M, Engberg S, Crovetto A, Vines L, Schou J, Hansen O. Monolithic thin-film chalcogenidesilicon tandem solar cells enabled by a diffusion barrier. Sol. Energy Mater. Sol. Cells 2020, 207: 110334.

[9] Moritz H, Futscher B E. Efficiency limit of perovskite/Si tandem solar cells. ACS Energy Lett. 2016, 1(4): 863868.

[10] Taguchi H, Soga T, Jimbo T. Fabrication of GaAs/Si tandem solar cell by epitaxial lift-off technique. Jpn. J. Appl. Phys. 2003, 42: 1419-1421.

[11] Kim B, Toprasertpong K, Paszuk A, Supplie O, Nakano Y, Hannappel T, Sugiyama M. GaAsP/Si tandem solar cells: Realistic prediction of efficiency gain by applying strain-balanced multiple quantum wells. Sol. Energy Mater. Sol. Cells 2018, 180: 303-310.

[12] Essig S, Steiner MA, Allebé C, Geisz JF, Paviet-Salomon B, Ward S, Descoeudres A, LaSalvia V, Barraud L, Badel N, Faes A, Levrat J, Despeisse M, Ballif C, Stradins P, Young DL. Realization of GaInP/Si dual-junction solar cells with $\mathbf{2 9 . 8 \%}$ 1-sun efficiency. IEEE J. Photovolt. 2016, 6(4): 1012-1019.

[13] He C, Han CB, Xu YR, Li XJ. Photovoltaic effect of CdS/Si nanoheterojunction array. J. Appl. Phys. 2011, 
110: 094316.

[14] Carmody M, Mallick S, Margetis J, Kodama R, Biegala T, Xu D, Bechmann P, Garland JW, Sivananthan S. Single-crystal II-VI on Si single-junction and tandem solar cells. Appl. Phys. Lett. 2010, 96: 153502,

[15] Leijtens T, Bush KA, Prasanna R, McGehee MD. Opportunities and challenges for tandem solar cells using metal halide perovskite semiconductors. Nat. Energy 2018, 3: 828-838 .

[16] Qiu ZW, Xu ZQ, Li NX, Zhou N, Chen YH, Wan XX, Liu JL, Li N, Hao XT, Bi PQ, Chen Q, Cao BQ, Zhou HP. Monolithic perovskite/Si tandem solar cells exceeding $22 \%$ efficiency via optimizing top cell absorber. Nano Energy, 2018, 53: 798-807.

[17] Jiang YJ, Almansouri I, Huang SJ, Young T, Li Y, Peng Y, Hou QC, Spiccia L, Bach U, Cheng YB, Green MA, Ho-Baillie A. Optical analysis of perovskite/silicon tandem solar cells. J. Mater. Chem. C 2016, 4: 5679-5689.

[18] Conibeer G, Perez-Wurfl I, Hao XJ, Di DW, Lin D. Si solid-state quantum dot-based materials for tandem solar cells. Nanoscale Res. Lett. 2012, 7: 193.

[19] Cheng QJ, Tam E, Xu SY, Ostrikov K. Si quantum dots embedded in an amorphous SiC matrix: nanophase control by non-equilibrium plasma hydrogenation. Nanoscale 2010, 2: 594-600.

[20] Heitmann J, Muller F, Zacharias M, Gosele U. Silicon nanocrystals: size matters. Adv. Mater. 2005, 17: 795 803.

[21] Uchida G, Yamamoto K, Sato M, Kawashima Y, Nakahara K, Kamataki K, Itagaki N, Koga K, Shiratani M. Effect of nitridation of Si nanoparticles on the performance of quantum-dot sensitized solar cells. Jpn. J. Appl. Phys. 2012, 51: $01 \mathrm{AD} 01$.

[22] Chang GR, Ma F, Ma DY, Xu KW. Multi-band silicon quantum dots embedded in an amorphous matrix of silicon carbide. Nanotechnology 2010, 21: 465605.

[23] Song DY, Cho EC, Conibeer G, Flynn C, Huang YD, Green MA. Structural, electrical and photovoltaic characterization of Si nanocrystals embedded SiC matrix and Si nanocrystals/c-Si heterojunction. Sol. Energy Mater. Sol. Cells 2008, 92: 474-481.

[24] Tsu R, Gonzalez-Hernandez J, Chao SS, Lee SC, Tanaka K. Critical volumefraction of crystallinity for conductivity percolation in phosphorus doped Si:F:H alloys. Appl. Phys. Lett. 1982, 40: 534-535.

[25] Campbell IH, Fauchet PM. The effects of microcrystal size and shape on the one phonon Raman spectra of crystalline semiconductors. Solid State Commun. 1986, 58: 739-741.

[26] Cao YQ, Xu X, Li SX, Li W, Xu J, Chen KJ. Improved photovoltaic properties of Si quantum dots/SiC multilayers-based heterojunction solar cells by reducing tunneling barrier thickness. Front. Optoelectron. 2013, 
6: $228-233$.

[27] Zhang P, Zhang XW, Xu J, Mu WW, Xu J, Li W, Chen KJ. Tunable nonlinear optical properties in nanocrystalline $\mathrm{Si} / \mathrm{SiO}_{2}$ multilayers under femtosecond excitation. Nanoscale Res. Lett. 2014, 9: 28.

[28] Wu W, Huang XF, Chen KJ, Xu JB, Gao X, Xu J, Li W. Room temperaturevisible electroluminescence in silicon nanostructures. J. Vac. Sci. Technol. A 1999, 17: 159-163.

[29] Rui YJ, Li SX, Cao YQ, Xu J, Li W, Chen KJ. Structural and electroluminescent properties of Si quantum dots/SiC multilayers. Appl. Surf. Sci. 2013, 269: 37-40.

[30] Priolo F, Gregorkiewicz T, Galli M, Krauss TF. Silicon nanostructures for photonics and photovoltaics. Nat. Nanotechnol. 2014, 9: 19-32.

[31] Rui YJ, Li SX, Xu J, Song C, Jiang XF, Li W, Chen KJ, Wang QM, Zuo YH. Size-dependent electroluminescence from Si quantum dots embedded in amorphous SiC matrix. J. Appl. Phys. 2011, 110: 064322 .

[32] Park S, Cho EC, Song DY, Conibeer G, Green MA. n-Type silicon quantum dots and p-type crystalline silicon heteroface solar cells. Sol. Energy Mater. Sol. Cells 2009, 93: 684-690.

[33] Boer K. Survey of Semiconductor Physics, van Nostrand Reinhold, New York, 1990, 244.

[34] Cao YQ, Lu P, Zhang XW, Xu J, Xu L, Chen KJ. Enhanced photovoltaic property by forming p-i-n structures containing Si quantum dots/SiC multilayers. Nanoscale Res. Lett. 2014, 9: 634.

[35] Tyagi MS, Van Overstraeten R. Minority carrier recombination in heavily-doped silicon. Solid-State Electron. 1983, 26: 577-597. 
Figure 1 Cross-sectional TEM images of as-deposited (a) a-Si (2nm)/a-SiC(2nm) MLs; (b) a-Si (4nm)/a-SiC(2nm) MLs; (c) a-Si (8nm)/a-SiC(2nm) MLs. The TEM images of annealed (d) Si QDs (2nm)/ SiC(2nm) MLs; (e) Si QDs (4nm)/ SiC(2nm) MLs; (f) Si QDs (8nm)/ SiC(2nm) MLs.

Figure 2 Raman spectra of $900^{\circ} \mathrm{C}$ annealed $\mathrm{Si}$ QDs/SiC MLs.

Figure 3 Optical absorption coefficient spectra of Si QDs/SiC MLs.

Figure 4 Dark current-voltage curves of solar cells containing different sized-Si QDs/SiC MLs.

Figure 5 AM1.5 illuminated current density-voltage curves of solar cells containing different sized-Si QDs/SiC MLs.

Figure 6 External quantum efficiency of solar cells containing different sized-Si QDs/SiC MLs.

Figure 7 External quantum efficiency of $\mathrm{p}^{+}$-i-n device structures containing different sized-Si QDs/SiC MLs.

Table 1 The device parameters of solar cells containing different sized-Si QDs/SiC MLs. 